\title{
Quantified trapezoidal fuzzy numbers
}

\author{
R. Adillon and L. Jorba \\ Departament de Matemàtica Econòmica, Financera i Actuarial, \\ Universitat de Barcelona, \\ Av. Diagonal, 690, 08034 Barcelona,Spain \\ adillon@ub.edu and lambert.jorba@ub.edu
}

\begin{abstract}
The aim of this work is to construct quantified trapezoidal fuzzy numbers as an extension of trapezoidal fuzzy numbers, by using modal intervals and accepting the possibility that the $\alpha$-cuts of a trapezoidal fuzzy number may also be improper intervals. In addition, this paper addresses the inclusion relationship which is deduced from the inclusion of modal intervals and is related to the classical setinclusion relationship between trapezoidal fuzzy numbers as well as the extensions of real continuous functions over the set of quantified trapezoidal fuzzy numbers.

Using the semantic interpretation of the calculations over modal intervals will enable us to interpret the meaning of the calculus accurately over quantified trapezoidal fuzzy numbers.

With quantified trapezoidal fuzzy numbers, we will be able to overcome some operational limitations that are usually faced when working with trapezoidal fuzzy numbers from a classical point of view. In order to show the applicability of quantified trapezoidal fuzzy numbers, we propose fuzzy equations which have no solution in the set of proper fuzzy numbers yet do have solutions that are improper fuzzy numbers. We also propose two applications of quantified trapezoidal fuzzy numbers, one of them about financial calculations and the other one in an optical problem.
\end{abstract}

keyword: Fuzzy numbers; Modal intervals; Semantic interpretation

\section{Introduction}

Whenever a numerical system presents deficiencies, it is advantageous to extend it to a new set so that those deficiencies are corrected while preserving the old properties, and to increase those properties if possible. Let us consider, for example, the operativity extension of the numerical sets which satisfy the inclusions: $\mathbb{N} \subseteq \mathbb{Z} \subseteq \mathbb{Q} \subseteq \mathbb{R} \subseteq \mathbb{C}$. Each new structure maintains and even increases the properties of the previous one.

The aim of some extensions of the real numbers is not (or not only) to achieve specific operativity, but on the basis of the real numbers, they are attempts to represent the numerical information from a more realistic point of view. Among these extensions we find the classical intervals which were first developed in the late 1950s by Moore [14] and the fuzzy numbers presented by Zadeh in 1965 [23]. 
Although is true that both structures contain the set of real numbers, neither maintains the whole set of properties of real numbers (thus, for instance: the distributive property is lost; the existence of a symmetric element with regard to the sum does not always make sense; and there is not always a symmetric element with regard to the product).

For example, the interval equation $[2,4]+X=[4,5]$ has no classical interval solution.

Some of the problems inherent to classical intervals are solved by their reticular completion using modal intervals [9].

Classical intervals do not solve the semantic interpretations which can be associated with an interval calculus; however, modal intervals do.

In this paper we use modal intervals to extend the concept of trapezoidal fuzzy numbers and obtain a new set of fuzzy numbers that we will call quantified trapezoidal fuzzy numbers. This extension will allow us to overcome some of the operative limitations on trapezoidal fuzzy numbers and to avoid the semantic ambiguity of fuzzy numbers.

\section{Preliminaries}

\subsection{Classical intervals and modal intervals}

An interval in its classical or traditional sense is defined as the set of real numbers between two bounds. The set of classical intervals is represented by $I(\mathbb{R})$; that is: $I(\mathbb{R})=\left\{\left[a_{1}, a_{2}\right]\right.$ such that $\left.a_{1} \leq a_{2}\right\}$.

In the field of classical interval analysis, we must highlight the studies by Moore [14], Nickel [15] and Alefeld [3], among others; and in the field of classical operations between intervals, we must mention Kaucher's arithmetic [13].

A modal interval is defined as a pair constructed by a classical interval and a quantifier. The set of modal intervals is represented by $I^{*}(\mathbb{R})$, that is $I^{*}(\mathbb{R})=$ $\left\{\left(\left[a_{1}, a_{2}\right], Q\right)\right.$ such that $\left.\left[a_{1}, a_{2}\right] \in I(\mathbb{R}), Q \in\{\forall, \exists\}\right\}$. The quantifier $Q$ associated with an interval in called the modal quantifier.

Given a modal interval $A=\left(\left[a_{1}, a_{2}\right], Q\right)$, we can define the substratum of $A$, which we represent as $\operatorname{set}(A)$, as $\operatorname{set}(A)=\left[a_{1}, a_{2}\right]$ and we can define the modality of $A$, which we represent as $\bmod (A)$, as $\bmod (A)=Q$. Modal intervals were introduced by E. Gardeñes [9].

Given a real predicate $P$ and given a modal interval $A=\left(\left[a_{1}, a_{2}\right], Q\right)$, we say that $P$ is a predicate accepted by the modal interval $A$, if $Q x \in\left[a_{1}, a_{2}\right], P(x)=1$.

A modal interval $A=\left(\left[a_{1}, a_{2}\right], Q\right)$ is identified with the set of real predicates accepted by $A$. This set of predicates accepted by $A$ is represented as $\operatorname{Pred}(A)$. Thus, a modal interval is a self-acceptor of real predicates.

There are two types of modal intervals: proper intervals and improper intervals. Proper intervals are those whose modal quantifier is $\exists$, while improper intervals are those whose modal quantifier is $\forall$. We represent the set of proper intervals as $I_{e}(\mathbb{R})$ and the set of improper intervals as $I_{u}(\mathbb{R})$. 


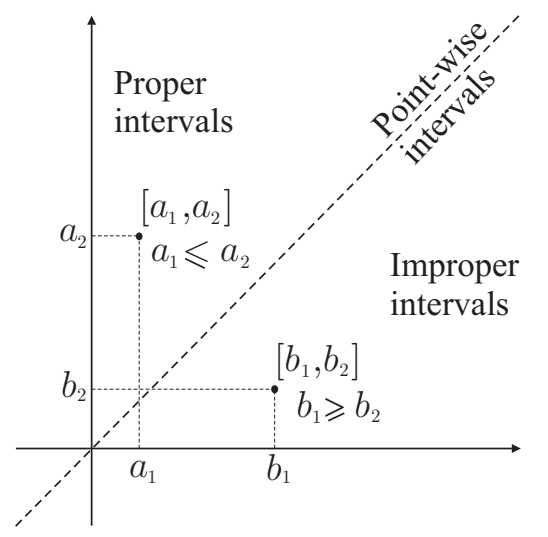

Figure 1: The interval plane.

If $A=\left(\left[a_{1}, a_{2}\right], Q\right)$ is a modal interval, we use the notation detailed in what follows, which allows us to make the modal quantifier implicit and considerably simplifies the writing. If $A$ is a proper interval, that is $A=\left(\left[a_{1}, a_{2}\right], \exists\right)$, then we denote it by $A=\left[a_{1}, a_{2}\right]$, identifying the proper intervals as the classical intervals. If $A$ is an improper interval, that is $A=\left(\left[a_{1}, a_{2}\right], \forall\right)$, then we denote it by $A=\left[a_{2}, a_{1}\right]$ with $a_{2} \geq a_{1}$.

Using this notation, the interval $[5,1]$ is the improper interval $([1,5], \forall)$. It is obvious that pointwise intervals can be considered as either proper or improper.

We will use the interval plane to represent proper and improper intervals, identifying the intervals with points in $\mathbb{R}^{2}$, as can be seen in Figure 1.

\subsection{Fuzzy numbers}

There are some tools to facilitate discussion of imprecision, vagueness and uncertainty, such as fuzzy sets and rough sets [16], [20], [24] among others. In this paper, we use the fuzzy numbers introduced by Zadeh [23] to construct quantified trapezoidal fuzzy numbers.

Let $X$ be a universal set, then a fuzzy set $A$ of $X$ is defined by its membership function $\mu_{A}: X \rightarrow[0,1]$, which assigns a real number $\mu_{A}(x)$ in the interval $[0,1]$ to each element $x \in X$. The value of $\mu_{A}(x)$ shows the degree to which $x$ is a member of $A$, or its degree of membership.

Given a fuzzy set $A$ of $X$ and given any real number $\alpha \in[0,1]$, the $\alpha$-cut of $A$ is a crisp set:

$$
A^{\alpha}= \begin{cases}\left\{x \in X \mid \mu_{A}(x) \geq \alpha\right\} & \text { if } \alpha \in(0,1], \\ \overline{\left\{x \in X \mid \mu_{A}(x)>0\right\}} & \text { if } \alpha=0,\end{cases}
$$

where $\overline{\left\{x \in X \mid \mu_{A}(x)>0\right\}}$ is the closure of the set $\left\{x \in X \mid \mu_{A}(x)>0\right\}$ and is called the support of $A$, which is denoted by $\operatorname{supp}(A)$. The $\alpha$-cut $A^{1}$ is called the core of $A$. 
In general (see for example, Dubois [5]), a fuzzy number $A$ is a fuzzy set $A$ of the real line $\mathbb{R}$ with membership function $\mu_{A}: \mathbb{R} \rightarrow[0,1]$ which is: normal (i.e., there exists an element $b \in \mathbb{R}$ such that $\mu_{A}(b)=1$ ); fuzzy convex (i.e., $\mu_{A}\left(\lambda x_{1}+(1-\lambda) x_{2}\right) \geq$ $\left.\mu_{A}\left(x_{1}\right) \wedge \mu_{A}\left(x_{2}\right) \forall x_{1}, x_{2} \in \mathbb{R}, \forall \lambda \in[0,1]\right)$; upper semi-continuous; and such that $\operatorname{supp}(A)$ is bounded.

We can describe the membership function of a fuzzy number as follows:

$$
\mu(x)= \begin{cases}f_{L}(x) & \text { if } a_{1} \leq x<a_{2}, \\ 1 & \text { if } a_{2} \leq x \leq a_{3}, \\ f_{U}(x) & \text { if } a_{3}<x \leq a_{4}, \\ 0 & \text { otherwise }\end{cases}
$$

where $a_{1}, a_{2}, a_{3}, a_{4}$ are real numbers such that $a_{1}<a_{2} \leq a_{3}<a_{4} ; f_{L}$ is a realvalued strictly increasing and right-continuous function; and $f_{U}$ is a real-valued strictly decreasing and left-continuous function.

The family $\left\{A^{\alpha} \mid \alpha \in[0,1]\right\}$ is a representation of the fuzzy number $A$.

A trapezoidal fuzzy number $A$ is defined by: $A=\left(a_{1}, a_{2}, a_{3}, a_{4}\right)$, where $a_{1}, a_{2}, a_{3}, a_{4} \in$ $\mathbb{R}, a_{1}<a_{2} \leq a_{3}<a_{4}$; and its membership function is defined as:

$$
\mu_{A}(x)= \begin{cases}\frac{x-a_{1}}{a_{2}-a_{1}} & \text { if } a_{1} \leq x<a_{2}, \\ 1 & \text { if } a_{2} \leq x \leq a_{3} \\ \frac{a_{4}-x}{a_{4}-a_{3}} & \text { if } a_{3}<x \leq a_{4}, \\ 0 & \text { otherwise }\end{cases}
$$

\section{Quantified trapezoidal fuzzy numbers}

This section explains how to construct quantified trapezoidal fuzzy numbers from the interval modal theory and the notion of classical trapezoidal fuzzy numbers.

Let $A=\left(a_{1}, a_{2}, a_{3}, a_{4}\right)$ be a trapezoidal fuzzy number. The support of $A$ (that is, the interval $\left.\left[a_{1}, a_{4}\right]\right)$ and the core of $A$ (the interval $\left[a_{2}, a_{3}\right]$ ) are traditional (classical) intervals. When we accept the possibility that both support and core are simultaneously improper intervals, then we have an opportunity to construct quantified trapezoidal fuzzy numbers.

Definition 1 A quantified trapezoidal fuzzy number $A$ is defined as: $A=\left(a_{1}, a_{2}, a_{3}, a_{4}\right)$, where $a_{1}, a_{2}, a_{3}, a_{4} \in \mathbb{R}$, satisfying $a_{1}<a_{2} \leq a_{3}<a_{4}$ or $a_{4}<a_{3} \leq a_{2}<a_{1}$; and its membership function is:

$$
\mu_{A}(x)= \begin{cases}\frac{x-a_{1}}{a_{2}-a_{1}} & \text { if } x \in \text { set }\left[a_{1}, a_{2}\right) \\ 1 & \text { if } x \in \text { set }\left[a_{2}, a_{3}\right] \\ \frac{x-a_{4}}{a_{3}-a_{4}} & \text { if } x \in \text { set }\left(a_{3}, a_{4}\right] \\ 0 & \text { otherwise }\end{cases}
$$

The difference between a trapezoidal fuzzy number and a quantified trapezoidal fuzzy number lies in the fact that there is no inequality restriction on the latter, either between the real values $a_{1}$ and $a_{4}$, or between the real values $a_{2}$ and $a_{3}$. 
The support of a quantified trapezoidal fuzzy number $A=\left(a_{1}, a_{2}, a_{3}, a_{4}\right)$ is the modal interval $\operatorname{supp}(A)=\left[a_{1}, a_{4}\right]$ and its core is the modal interval core $(A)=\left[a_{2}, a_{3}\right]$.

It can easily proven that any quantified trapezoidal fuzzy number $A$ satisfies: $\operatorname{set}(\operatorname{core}(A)) \subseteq$ $\operatorname{set}(\operatorname{supp}(A))$.

Definition 2 A quantified trapezoidal fuzzy number $A$ is proper if both the core and support of $A$ are proper intervals; while the quantified trapezoidal fuzzy number is improper if both the core and support of A are improper intervals.

Definition 3 Given a quantified trapezoidal fuzzy number $A=\left(a_{1}, a_{2}, a_{3}, a_{4}\right)$, we define the set of $A$, set $(A)$, as the trapezoidal fuzzy number in its classical sense: $\operatorname{set}(A) \quad=\quad\left(\min \left\{a_{1}, a_{4}\right\}, \min \left\{a_{2}, a_{3}\right\}\right.$, $\left.\max \left\{a_{2}, a_{3}\right\}, \max \left\{a_{1}, a_{4}\right\}\right)$.

Definition 4 Given a quantified trapezoidal fuzzy number $A=\left(a_{1}, a_{2}, a_{3}, a_{4}\right)$, we define the modality of $A, \bmod (A)$, as the interval modality of the core and the support of $A$; that is, $\bmod (A)=\bmod \left(\left[a_{1}, a_{4}\right]\right)=\bmod \left(\left[a_{2}, a_{3}\right]\right)$.

Using these definitions (Definitions 1, 2, 3 and 4), since a modal interval consists of a set and a quantifier, it follows that a quantified trapezoidal fuzzy number is a pair formed by a trapezoidal fuzzy number in its traditional sense, and a quantifier.

Thus, a quantified trapezoidal fuzzy number $A=\left(a_{1}, a_{2}, a_{3}, a_{4}\right)$ is:

$$
A=\left(\operatorname{set}\left(a_{1}, a_{2}, a_{3}, a_{4}\right), Q\right)
$$

where $\left[a_{1}, a_{4}\right],\left[a_{2}, a_{3}\right] \in I^{*}(\mathbb{R}), \operatorname{set}\left(\left[a_{2}, a_{3}\right]\right) \subseteq \operatorname{set}\left(\left[a_{1}, a_{4}\right]\right)$ and $Q \in\{\exists, \forall\}$.

If the quantified trapezoidal fuzzy number $A$ is proper:

$$
A=\left(\left(a_{1}, a_{2}, a_{3}, a_{4}\right), \exists\right)
$$

and the intervals $\left[a_{1}, a_{4}\right]$ and $\left[a_{2}, a_{3}\right]$ are proper intervals, then it follows that: $a_{1} \leq a_{4}$ and $a_{2} \leq a_{3}$. Thus, $\operatorname{set}\left(a_{1}, a_{2}, a_{3}, a_{4}\right)=\left(a_{1}, a_{2}, a_{3}, a_{4}\right)$.

In contrast, if the quantified trapezoidal fuzzy number $A$ is improper:

$$
A=\left(\left(a_{4}, a_{3}, a_{2}, a_{1}\right), \forall\right)
$$

because the intervals $\left[a_{1}, a_{4}\right]$ and $\left[a_{2}, a_{3}\right]$ are improper intervals, so it follows that $a_{4} \leq$ $a_{1}$ and $a_{3} \leq a_{2}$. Thus, $\operatorname{set}\left(a_{1}, a_{2}, a_{3}, a_{4}\right)=\left(a_{4}, a_{3}, a_{2}, a_{1}\right)$.

The notation used in modal interval theory [17] allows us to use the following canonical notation for quantified trapezoidal fuzzy numbers:

$$
\begin{aligned}
& A=\left(a_{1}, a_{2}, a_{3}, a_{4}\right) \text { is proper if } a_{1} \leq a_{2} \leq a_{3} \leq a_{4}, \\
& A=\left(a_{1}, a_{2}, a_{3}, a_{4}\right) \text { is improper if } a_{1} \geq a_{2} \geq a_{3} \geq a_{4} .
\end{aligned}
$$

We will refer to the set of quantified trapezoidal fuzzy numbers as $T^{*}(\mathbb{R})$.

Proposition 5 Given a quantified trapezoidal fuzzy number $A=\left(a_{1}, a_{2}, a_{3}, a_{4}\right)$, the modality of the $\alpha$-cuts of $A$ is the same as the modality of the quantified fuzzy number A. 


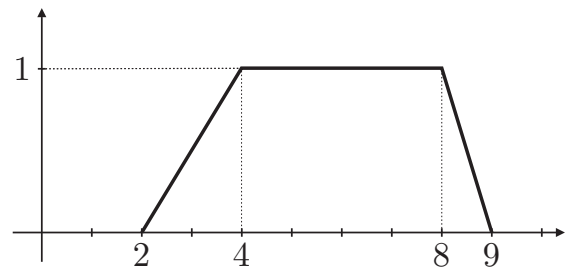

Figure 2: Classical representation of a trapezoidal fuzzy number.

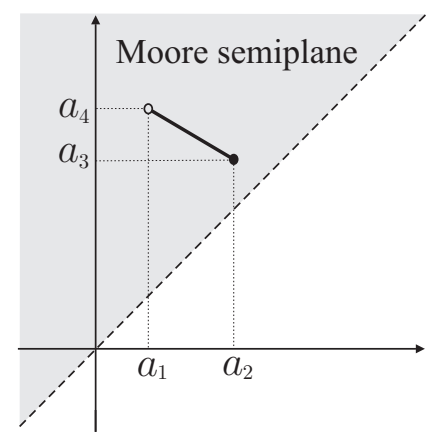

Figure 3: Representation in the Moore semi-plane of a classical trapezoidal fuzzy number.

Proof. For a given $\alpha \in[0,1]$ the $\alpha$-cut of $A$ is:

$$
\begin{aligned}
A^{\alpha} & =(1-\alpha)\left[a_{1}, a_{4}\right]+\alpha\left[a_{2}, a_{3}\right]= \\
& =\left[(1-\alpha) a_{1}+\alpha a_{2},(1-\alpha) a_{4}+\alpha a_{3}\right] .
\end{aligned}
$$

If $A$ is proper, $a_{1} \leq a_{4}$ and $a_{2} \leq a_{3}$. As $\alpha \in[0,1],(1-\alpha) a_{1}+\alpha a_{2} \leq$ $(1-\alpha) a_{4}+\alpha a_{3}$, which means that $A^{\alpha}$ is proper.

If $A$ is improper, $a_{1} \geq a_{4}$ and $a_{2} \geq a_{3}$. As $\alpha \in[0,1],(1-\alpha) a_{1}+\alpha a_{2} \geq$ $(1-\alpha) a_{4}+\alpha a_{3}$, which means that $A^{\alpha}$ is improper.

The modality of quantified trapezoidal fuzzy numbers results in serious limitations when representing their membership function graphically in the traditional plane. Thus, the representation in Figure 2 does not contain enough information to determine whether we are referring to the proper trapezoidal fuzzy number $(2,4,8,9)$ or to the improper one $(9,8,4,2)$.

This is the reason why we represent quantified fuzzy numbers graphically in the interval plane. This representation offers us the opportunity to distinguish proper trapezoidal fuzzy numbers from improper ones (See [2]).

Given a classical trapezoidal fuzzy number $A=\left(a_{1}, a_{2}, a_{3}, a_{4}\right)$, its graphical representation in the Moore semi-plane is shown in Figure 3.

In this representation (Figure 3), the symbol " $\bullet$ " refers to the core of the fuzzy number; while the symbol "०" refers to the support of the fuzzy number. 


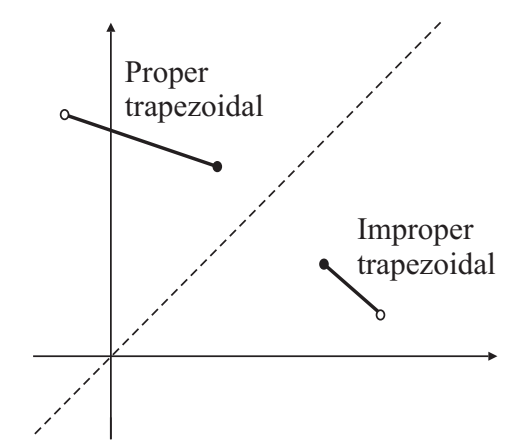

Figure 4: Representation of a quantified trapezoidal fuzzy number in the interval plane.

For each $\alpha \in[0,1]$, the point $\alpha\left(a_{2}, a_{3}\right)+(1-\alpha)\left(a_{1}, a_{4}\right)$ corresponds to the $\alpha$-cut, $A^{\alpha}$, of the trapezoidal fuzzy number $A=\left(a_{1}, a_{2}, a_{3}, a_{4}\right)$ [2, Proposition 1].

At the same time, any trapezoidal fuzzy number is determined by a decreasing segment in the Moore semi-plane.

If both the core and support of $A$ can be simultaneously improper intervals, then the Moore semi-plane it is not enough to represent them graphically and we must extend the representation to the interval plane. This expands the set of intervals that can be represented graphically and allows us to represent improper intervals.

When representing a quantified trapezoidal fuzzy number, there are two different possibilities, as can be seen in Figure 4.

As both the core and support of the quantified trapezoidal fuzzy number have the same modality, the graphical representation of a proper trapezoidal fuzzy number in the interval plane will be placed above the straight line $y=x$; while the graphical representation of an improper trapezoidal fuzzy number in the interval plane will be placed below it.

When the core of a quantified trapezoidal fuzzy number is a pointwise interval, we can refer to it as a triangular modal fuzzy number, as the core can be considered either proper or improper. In this case, the core will always be placed on the straight line $y=x$.

When representing a quantified trapezoidal fuzzy number $A=\left(a_{1}, a_{2}, a_{3}, a_{4}\right)$ in the interval plane, for each $\alpha \in[0,1]$, the point in $\mathbb{R}^{2} \alpha\left(a_{2}, a_{3}\right)+(1-\alpha)\left(a_{1}, a_{4}\right)$ corresponds to the $\alpha$-cut of $A$. Consequently, it follows that:

$$
\forall \alpha \in[0,1], \bmod \left(A^{\alpha}\right)=\bmod (A)
$$

Now we are going to define some operators in $T^{*}(\mathbb{R})$ related to the interval modality and we are also going to focus on the inclusion relationship over the set $T^{*}(\mathbb{R})$, which will show the existence of the infimum and supremum (meet and join) with regard to the inclusion relationship; which means that $\left(T^{*}(\mathbb{R}), \subseteq\right)$ is a reticle.

In the set of quantified trapezoidal fuzzy numbers, $T^{*}(\mathbb{R})$, the following modal operators are defined: 
Dual operator. If $A=\left(a_{1}, a_{2}, a_{3}, a_{4}\right) \in T^{*}(\mathbb{R})$, the dual operator is defined as:

$$
\operatorname{dual}(A)=\left(a_{4}, a_{3}, a_{2}, a_{1}\right) \in T^{*}(\mathbb{R})
$$

Proper operator. If $A=\left(a_{1}, a_{2}, a_{3}, a_{4}\right) \in T^{*}(\mathbb{R})$, the proper operator is defined as:

$$
\begin{aligned}
\operatorname{prop}(A)= & \left(\min \left\{a_{1}, a_{4}\right\}, \min \left\{a_{2}, a_{3}\right\},\right. \\
& \left.\max \left\{a_{2}, a_{3}\right\}, \max \left\{a_{1}, a_{4}\right\}\right) \in T^{*}(\mathbb{R})
\end{aligned}
$$

Improper operator. If $A=\left(a_{1}, a_{2}, a_{3}, a_{4}\right) \in T^{*}(\mathbb{R})$, the improper operator is defined as:

$$
\begin{aligned}
\operatorname{impr}(A)= & \left(\max \left\{a_{1}, a_{4}\right\}, \max \left\{a_{2}, a_{3}\right\},\right. \\
& \left.\min \left\{a_{2}, a_{3}\right\}, \min \left\{a_{1}, a_{4}\right\}\right) \in T^{*}(\mathbb{R})
\end{aligned}
$$

The inclusion relation is significant when interpreting a calculus involving quantified trapezoidal fuzzy numbers semantically. The inclusion relation must be defined from the modal interval inclusion of the $\alpha$-cuts, which will determine the semantic interpretation.

Given the modal intervals $A$ and $B$, the inclusion between $A$ and $B$ is defined from the inclusion of the set of predicates accepted for each one of these intervals (see [9], [17]); that is:

$$
A \subseteq B \text { if Pred }(A) \subseteq \operatorname{Pred}(B)
$$

Using the canonical notation, if $A=\left[a_{1}, a_{2}\right]$ and $B=\left[b_{1}, b_{2}\right]$ are modal intervals, the inclusion relation can be expressed in its classical form:

$$
\left[a_{1}, a_{2}\right] \subseteq\left[b_{1}, b_{2}\right] \text { if } a_{1} \geq b_{1} \text { and } a_{2} \leq b_{2}
$$

The set of classical intervals $I(\mathbb{R})$ is not a reticle for the inclusion relation, while the set of modal intervals, $I^{*}(\mathbb{R})$ is.

Definition 6 Given $A, B \in T^{*}(\mathbb{R})$, we define $A \subseteq B$ if $\forall \alpha \in[0,1] A^{\alpha} \subseteq B^{\alpha}$, where this second inclusion refers to the interval modal inclusion.

Proposition 7 Given $A, B \in T^{*}(\mathbb{R})$, where $A=\left(a_{1}, a_{2}, a_{3}, a_{4}\right)$ and $B=\left(b_{1}, b_{2}, b_{3}, b_{4}\right)$, it follows that:

1. If $A$ and $B$ are proper, that is $\bmod (A)=\bmod (B)=\exists$, then:

$$
\begin{aligned}
A \subseteq B \Leftrightarrow & \operatorname{set}(\operatorname{supp}(A)) \subseteq \operatorname{set}(\operatorname{supp}(B)) \\
& \text { and } \\
& \operatorname{set}(\operatorname{core}(A)) \subseteq \operatorname{set}(\operatorname{core}(B)),
\end{aligned}
$$

2. If $A$ and $B$ are improper, that is $\bmod (A)=\bmod (B)=\forall$, then:

$$
\begin{aligned}
A \subseteq B \Leftrightarrow & \operatorname{set}(\operatorname{supp}(A)) \supseteq \operatorname{set}(\operatorname{supp}(B)) \\
& \text { and } \\
& \operatorname{set}(\operatorname{core}(A)) \supseteq \operatorname{set}(\operatorname{core}(B)),
\end{aligned}
$$


3. If $A$ is proper and $B$ improper, that is $\bmod (A)=\exists, \bmod (B)=\forall$, then:

$$
A \subseteq B \Leftrightarrow A=B=(p, p, p, p), p \in \mathbb{R},
$$

4. If $A$ is improper and $B$ proper, that is $\bmod (A)=\forall, \bmod (B)=\exists$, then:

$$
A \subseteq B \Leftrightarrow \operatorname{set}(\operatorname{core}(A)) \cap \operatorname{set}(\operatorname{core}(B)) \neq \emptyset .
$$

\section{Proof.}

1. $\Rightarrow) A \subseteq B \Rightarrow A^{0} \subseteq B^{0} \Leftrightarrow \operatorname{supp}(A) \subseteq \operatorname{supp}(B)$. As both $\operatorname{supp}(A)$ and $\operatorname{supp}(B)$ are proper intervals, $\operatorname{set}(\operatorname{supp}(A))=\operatorname{supp}(A)$ and $\operatorname{set}(\operatorname{supp}(B))=$ $\operatorname{supp}(B)$. Analogously, if $\alpha=1$, we obtain the interval inclusion set $($ core $(A)) \subseteq$ $\operatorname{set}(\operatorname{core}(B))$.

$\Leftarrow) \operatorname{set}(\operatorname{supp}(A))=\left[a_{1}, a_{4}\right], \operatorname{set}(\operatorname{supp}(B))=\left[b_{1}, b_{4}\right]$, set $(\operatorname{core}(A))=$ $\left[a_{2}, a_{3}\right]$ and

set $($ core $(B))=\left[b_{2}, b_{3}\right]$. As $\left[a_{1}, a_{4}\right] \subseteq\left[b_{1}, b_{4}\right]$, it follows $a_{1} \geq b_{1}$ and $a_{4} \leq b_{4}$. As $\left[a_{2}, a_{3}\right] \subseteq\left[b_{2}, b_{3}\right]$, then $a_{2} \geq b_{2}$ and $a_{3} \leq b_{3}$ and so, for any $\alpha \in[0,1]$, $(1-\alpha) a_{1}+\alpha a_{2} \geq(1-\alpha) b_{1}+\alpha b_{2}$ and $(1-\alpha) a_{4}+\alpha a_{3} \leq(1-\alpha) b_{4}+\alpha b_{3}$. This means $\forall \alpha \in[0,1], A^{\alpha} \subseteq B^{\alpha}$.

2. $\Rightarrow) A \subseteq B \Rightarrow A^{0} \subseteq B^{0} \Leftrightarrow\left[a_{1}, a_{4}\right] \subseteq\left[b_{1}, b_{4}\right]$. As both are improper intervals, using the interval modal inclusion, $\left[a_{1}, a_{4}\right] \subseteq\left[b_{1}, b_{4}\right] \Leftrightarrow \operatorname{set}\left(\left[a_{1}, a_{4}\right]\right) \supseteq$ $\operatorname{set}\left(\left[b_{1}, b_{4}\right]\right) \Leftrightarrow \operatorname{set}(\operatorname{supp}(A)) \supseteq \operatorname{set}(\operatorname{supp}(B))$. From the relation $A \subseteq B$, we also have $A^{1} \subseteq B^{1}$, and reasoning similar to that just given, we obtain $\operatorname{set}(\operatorname{core}(A)) \supseteq \operatorname{set}(\operatorname{core}(B))$.

$\Leftarrow) \operatorname{set}(\operatorname{supp}(A)) \supseteq \operatorname{set}(\operatorname{supp}(B)) \Leftrightarrow\left[a_{4}, a_{1}\right] \supseteq\left[b_{4}, b_{1}\right] \Leftrightarrow a_{4} \leq b_{4}$ and $a_{1} \geq b_{1}$. Moreover, set $($ core $(A)) \supseteq \operatorname{set}($ core $(B)) \Leftrightarrow\left[a_{3}, a_{2}\right] \supseteq\left[b_{3}, b_{2}\right] \Leftrightarrow$ $a_{3} \leq b_{3}$ and $a_{2} \geq b_{2}$. For any $\alpha \in[0,1],(1-\alpha) a_{1}+\alpha a_{2} \geq(1-\alpha) b_{1}+\alpha b_{2}$ and $(1-\alpha) a_{4}+\alpha a_{3} \leq(1-\alpha) b_{4}+\alpha b_{3} \Rightarrow \forall \alpha \in[0,1], A^{\alpha} \subseteq B^{\alpha}$.

3. $\Rightarrow) A^{0}=\left[a_{1}, a_{4}\right]$ is proper; $B^{0}=\left[b_{1}, b_{4}\right]$ is improper. The modal interval inclusion $A^{0} \subseteq B^{0}$ becomes $A^{0}=B^{0}$. Moreover, these two intervals are pointwise intervals; that is, $A^{0}=B^{0}=[p, p]$. The same reasoning applied to the proper interval $A^{1}=\left[a_{2}, a_{3}\right]$ and to the improper interval $B^{1}=\left[b_{2}, b_{3}\right]$, leads us to the same result; and so $A=B=(p, p, p, p)$.

$\Leftarrow)$ Trivially.

4. $\Rightarrow)$ Taking $\alpha=1, A^{1}=\operatorname{core}(A), B^{1}=\operatorname{core}(B)$, as $A^{1}$ is an improper interval, $B^{1}$ is a proper interval and $A^{1} \subseteq B^{1}$, using the modal interval inclusion we obtain set $($ core $(A)) \cap \operatorname{set}($ core $(B)) \neq \emptyset$.

$\Leftarrow) \forall \alpha \in[0,1]$, set $(\operatorname{core}(A)) \subseteq \operatorname{set}\left(A^{\alpha}\right)$, set $(\operatorname{core}(B)) \subseteq \operatorname{set}\left(B^{\alpha}\right)$ and set $(\operatorname{core}(A)) \cap \operatorname{set}(\operatorname{core}(B)) \neq \emptyset$, which means $\exists p$ such that $p \in \operatorname{set}(\operatorname{core}(A)) \cap$ $\operatorname{set}(\operatorname{core}(B))$. That is $p \in \operatorname{set}\left(A^{\alpha}\right) \cap \operatorname{set}\left(B^{\alpha}\right) \Rightarrow \operatorname{set}\left(A^{\alpha}\right) \cap \operatorname{set}\left(B^{\alpha}\right) \neq \emptyset$.

Because of the modality of $A^{\alpha}$ and $B^{\alpha}$ it holds that $A^{\alpha} \subseteq B^{\alpha}$, and so $A \subseteq B$. 
Proposition 8 Given $A, B \in T^{*}(\mathbb{R}), A=\left(a_{1}, a_{2}, a_{3}, a_{4}\right)$ and $B=\left(b_{1}, b_{2}, b_{3}, b_{4}\right)$, using the interval canonical notation it holds that:

$$
A \subseteq B \Leftrightarrow a_{1} \geq b_{1}, a_{4} \leq b_{4} \text { and } a_{2} \geq b_{2}, a_{3} \leq b_{3}
$$

Proof. Using the canonical notation for modal intervals and the expression for the inclusion of quantified trapezoidal fuzzy numbers studied in the above Proposition 7.

Definition 9 Given $A, B \in T^{*}(\mathbb{R})$, we define:

1. meet $(A, B)$ which is represented by $A \wedge B$ as the quantified trapezoidal fuzzy number which satisfies:

- $A \wedge B \subseteq A$ and $A \wedge B \subseteq B$.

- If $C \in T^{*}(\mathbb{R})$ is such that $C \subseteq A$ and $C \subseteq B$, then $C \subseteq A \wedge B$;

2. join $(A, B)$ which is represented by $A \vee B$ as the quantified trapezoidal fuzzy number which satisfies:

- $A \subseteq A \vee B$ and $B \subseteq A \vee B$.

- If $C \in T^{*}(\mathbb{R})$ is such that $A \subseteq C$ and $B \subseteq C$, then $A \vee B \subseteq C$.

The meet and join of two quantified trapezoidal fuzzy numbers correspond to the supremum and to the infimum with regard to the inclusion relation.

Proposition 10 If $A, B \in T^{*}(\mathbb{R}), A=\left(a_{1}, a_{2}, a_{3}, a_{4}\right)$ and $B=\left(b_{1}, b_{2}, b_{3}, b_{4}\right)$, then:

(a)

$$
\begin{aligned}
A \wedge B= & \left(\max \left\{a_{1}, b_{1}\right\}, \max \left\{a_{2}, b_{2}\right\},\right. \\
& \left.\min \left\{a_{3}, b_{3}\right\}, \min \left\{a_{4}, b_{4}\right\}\right) \in T^{*}(\mathbb{R}),
\end{aligned}
$$

(b)

$$
\begin{aligned}
A \vee B= & \left(\min \left\{a_{1}, b_{1}\right\}, \min \left\{a_{2}, b_{2}\right\},\right. \\
& \left.\max \left\{a_{3}, b_{3}\right\}, \max \left\{a_{4}, b_{4}\right\}\right) \in T^{*}(\mathbb{R}) .
\end{aligned}
$$

Proof. The $\alpha$-cuts of $A$ and $B$ are $A^{\alpha}=\alpha\left[a_{2}, a_{3}\right]+(1-\alpha)\left[a_{1}, a_{4}\right]$ and $B^{\alpha}=$ $\alpha\left[b_{2}, b_{3}\right]+(1-\alpha)\left[b_{1}, b_{4}\right]$.

(a) Let $C=\left(\max \left\{a_{1}, b_{1}\right\}, \max \left\{a_{2}, b_{2}\right\}\right.$, $\left.\min \left\{a_{3}, b_{3}\right\}, \min \left\{a_{4}, b_{4}\right\}\right)$. The $\alpha$-cuts of $C$ are

$$
\begin{aligned}
C^{\alpha}= & {\left[\alpha \max \left\{a_{2}, b_{2}\right\}+(1-\alpha) \max \left\{a_{1}, b_{1}\right\},\right.} \\
& \left.\alpha \min \left\{a_{3}, b_{3}\right\}+(1-\alpha) \min \left\{a_{4}, b_{4}\right\}\right]
\end{aligned}
$$


It holds that $\forall \alpha \in[0,1], C^{\alpha} \subseteq A^{\alpha}$, because:

$$
\begin{gathered}
\alpha \max \left\{a_{2}, b_{2}\right\}+(1-\alpha) \max \left\{a_{1}, b_{1}\right\} \geq \\
\alpha a_{2}+(1-\alpha) a_{1} \\
\alpha \min \left\{a_{3}, b_{3}\right\}+(1-\alpha) \min \left\{a_{4}, b_{4}\right\} \leq \\
\alpha a_{3}+(1-\alpha) a_{4}
\end{gathered}
$$

In the same way, $C^{\alpha} \subseteq B^{\alpha}$. From which it follows that $C \subseteq A$ and $C \subseteq B$.

Moreover, if $X=\left(x_{1}, x_{2}, x_{3}, x_{4}\right)$ satisfies $X \subseteq A$ and $X \subseteq B$, it holds that:

$$
\forall \alpha \in[0,1] X^{\alpha} \subseteq A^{\alpha} \text { and } X^{\alpha} \subseteq B^{\alpha} .
$$

From $X^{\alpha} \subseteq A^{\alpha}$ we obtain:

$$
\begin{aligned}
& \alpha x_{2}+(1-\alpha) x_{1} \geq \alpha a_{2}+(1-\alpha) a_{1} \\
& \alpha x_{3}+(1-\alpha) x_{4} \leq \alpha a_{3}+(1-\alpha) a_{4}
\end{aligned}
$$

and $x_{1} \geq a_{1}, x_{2} \geq a_{2}, x_{3} \leq a_{3}, x_{4} \leq a_{4}$.

In the same way, from $X^{\alpha} \subseteq B^{\alpha}$ we obtain $x_{1} \geq b_{1}, x_{2} \geq b_{2}, x_{3} \leq b_{3}, x_{4} \leq b_{4}$. It is obvious that $x_{1} \geq \max \left\{a_{1}, b_{1}\right\}, x_{2} \geq \max \left\{a_{2}, b_{2}\right\}, x_{3} \leq \min \left\{a_{3}, b_{3}\right\}$ and $x_{4} \leq \min \left\{a_{4}, b_{4}\right\}$; and so it follows that $X \subseteq C$.

Thus, $C=A \wedge B$.

(b) As the proof of (a) above.

Corollary 11 Under the conditions of the above Proposition 10, if both $A$ and $B$ have the same modality, then $A \wedge B$ and $A \vee B$ also have this same modality. If the modalities of $A$ and $B$ are not the same, then the modality of $A \wedge B$ is improper, while the modality of $A \vee B$ is proper.

\section{Fuzzy modal extensions and semantics}

When working with mathematical objects which represent fuzziness or imprecision, such as fuzzy numbers or intervals, we must bear in mind that in any calculus it is very important to know the meaning of what we have evaluated; that is, the semantic interpretation of the result obtained.

Let $A_{1}, \ldots, A_{n}$ be trapezoidal fuzzy numbers in their traditional sense, and let $f$ be a rational function. If $B$ is the exact solution of the calculus $f\left(A_{1}, \ldots, A_{n}\right)$, then we can provide an interpretation of this calculus using the $\alpha$-cuts [8] and we obtain that $\forall \alpha \in[0,1]$ satisfies:

$$
\left(\forall a_{1} \in A_{1}^{\alpha}\right) \cdots\left(\forall a_{n} \in A_{n}^{\alpha}\right)\left(\exists b \in B^{\alpha}\right)
$$


such that $b=f\left(a_{1}, \ldots a_{n}\right)$;

or instead:

$$
\left(\forall b \in B^{\alpha}\right)\left(\exists a_{1} \in A_{1}^{\alpha}\right) \cdots\left(\exists a_{n} \in A_{n}^{\alpha}\right)
$$

such that $b=f\left(a_{1}, \ldots a_{n}\right)$.

The exact value $f\left(A_{1}, \ldots, A_{n}\right)=B$ is usually difficult to evaluate and in most cases we replace every rational operator in the function $f$ by its corresponding interval operator. The result of these substitutions will be a fuzzy number $Z$ such that:

$$
f\left(A_{1}, \ldots, A_{n}\right) \subseteq Z
$$

and there will only be one valid semantic interpretation deduced from (3), and it will be, $\forall \alpha \in[0,1]$ :

$$
\left(\forall a_{1} \in A_{1}^{\alpha}\right) \cdots\left(\forall a_{n} \in A_{n}^{\alpha}\right)\left(\exists z \in Z^{\alpha}\right)
$$

such that $z=f\left(a_{1}, \ldots, a_{n}\right)$.

However, the fuzzy number $Z$ will not, in general, be a trapezoidal fuzzy number. If we need the result to be trapezoidal and we want to maintain the semantic interpretation, it will be necessary to find a trapezoidal fuzzy number, $Y$, such that $Z \subseteq Y$; then we will be able to guarantee that, $\forall \alpha \in[0,1]$ :

$$
\left(\forall a_{1} \in A_{1}^{\alpha}\right) \cdots\left(\forall a_{n} \in A_{n}^{\alpha}\right)\left(\exists y \in Y^{\alpha}\right)
$$

such that $y=f\left(a_{1}, \ldots, a_{n}\right)$.

The situation described above is inherent to the calculus with trapezoidal fuzzy numbers and is one of the handicaps we find when working with them. This situation becomes obvious when we multiply two trapezoidal fuzzy numbers, which means that not all the basic operators are internal operations in the set of trapezoidal fuzzy numbers.

To move operatively close to these calculations on the set of trapezoidal fuzzy numbers, we can apply some criteria which bring a non-trapezoidal result near to a trapezoidal result. Fuzzy number approximations have been studied by many researchers. Abbasbandy and Asady [1] introduced a fuzzy trapezoidal approximation, using the metric distance between two fuzzy numbers. Grzegorzewski and Mrówka [10], and Grzegorzewski [11] studied a nearest trapezoidal approximation operator preserving the expected interval. Yeh [21], [22] studied an improvement to the nearest trapezoidal approximation operator preserving the expected interval. Veerani et al. [18] proposed a method to convert any fuzzy number to the nearest symmetric trapezoidal fuzzy number approximation preserving the expected interval. Ban, Coroianu and Khastan [4] developed a general method to study the LâĂŞR approximations of fuzzy numbers preserving ambiguity, value and width. Recently some methods for ranking fuzzy numbers using distances have been developed [12], [6].

None of those trapezoidal approximations is useful to us, because although they preserve certain properties, such as the expected interval, they do not impose preservation of inclusivity and so they are not valid for semantic interpretations.

In the work we present in this paper, it is essential to evaluate the result of the calculation that yields the trapezoidal $Y$ containing the fuzzy number $Z$. We require 
that the trapezoidal fuzzy number $Y$ is an optimal inclusion of $Z$, in the sense studied by Wagen [19].

Let us now define the $*$-semantic extension on a set of quantified trapezoidal fuzzy numbers, using the interval $*$-semantic extension of a real continuous function.

Definition 12 ([17, Definition 3.2.1]) Let $f: \mathbb{R}^{n} \rightarrow \mathbb{R}$ be a real continuous function and $x=\left(x_{p}, x_{i}\right)$ the component splitter corresponding to $X=\left(X_{p}, X_{i}\right) \in I^{*}\left(\mathbb{R}^{n}\right)$, where $X_{p}$ are the proper interval components of $X$, and $X_{i}$ are the improper interval components of $X$. The $*$-interval extension of $f$ is represented by $f^{*}$ and is defined as:

$$
f^{*}(X)=\underset{x_{p} \in \operatorname{set}\left(X_{p}\right)}{\vee} \underset{x_{i} \in \operatorname{set}\left(X_{i}\right)}{\wedge}\left[f\left(x_{p}, x_{i}\right), f\left(x_{p}, x_{i}\right)\right]
$$

Definition 13 The $*$-fuzzy extension of a real continuous function $f: \mathbb{R}^{n} \rightarrow \mathbb{R}$, over the quantified trapezoidal fuzzy numbers $X_{1}, \ldots, X_{n}$, is represented by $T f^{*}\left(X_{1}, \ldots, X_{n}\right)$ and is evaluated using the interval extension $f^{*}$ above the $\alpha$-cuts $X_{1}^{\alpha}, \ldots, X_{n}^{\alpha}$. If $X_{i}=\left\{X_{i}^{\alpha}, \alpha \in[0,1]\right\}$, then:

$T f^{*}\left(X_{1}, \ldots, X_{n}\right)=\left\{\left(f^{*}\left(X_{1}^{\alpha}, \ldots, X_{n}^{\alpha}\right)\right)^{\alpha}, \alpha \in[0,1]\right\}$ that is

$$
\begin{aligned}
& \operatorname{Tf}^{*}\left(X_{1}, \ldots, X_{n}\right)= \\
& \quad\left\{\left[\min _{x_{p} \in \operatorname{set}\left(X_{p}^{\alpha}\right)} \max _{x_{i} \in \operatorname{set}\left(X_{i}^{\alpha}\right)} f\left(x_{p}, x_{i}\right),\right.\right. \\
& \left.\left.\quad \max _{x_{p} \in \operatorname{set}\left(X_{p}^{\alpha}\right)} \min _{x_{i} \in \operatorname{set}\left(X_{i}^{\alpha}\right)} f\left(x_{p}, x_{i}\right)\right]^{\alpha}, \alpha \in[0,1]\right\}
\end{aligned}
$$

which is equivalent to:

$$
\begin{aligned}
& T f^{*}\left(X_{1}, \ldots, X_{n}\right)= \\
& \quad\left\{\underset{x_{p} \in \operatorname{set}\left(X_{p}^{\alpha}\right)}{\vee} x_{i} \in \operatorname{set}\left(X_{i}^{\alpha}\right)\right. \\
& \left.\left.\left.\quad f\left(x_{p}, x_{i}\right)\right]\right)^{\alpha}, \alpha \in[0,1]\right\} .
\end{aligned}
$$

Proposition 14 Given a continuous real function $f: \mathbb{R}^{n} \rightarrow \mathbb{R}$ and $X_{1}, \ldots, X_{n} \in$ $T^{*}(\mathbb{R})$, the calculus $T f^{*}\left(X_{1}, \ldots, X_{n}\right)$ verifies: if $\alpha, \beta \in[0,1]$ and $\alpha \geq \beta$, then $\left(T f^{*}\left(X_{1}, \ldots, X_{n}\right)\right)^{\alpha} \subseteq\left(T f^{*}\left(X_{1}, \ldots, X_{n}\right)\right)^{\beta}$.

Proof. Let $\alpha, \beta \in[0,1]$. If $\alpha \geq \beta$, then $X_{1}^{\alpha} \subseteq X_{1}^{\beta}, \ldots, X_{n}^{\alpha} \subseteq X_{n}^{\beta}$.

As $\left(T f^{*}\left(X_{1}, \ldots, X_{n}\right)\right)^{\alpha}=f^{*}\left(X_{1}^{\alpha}, \ldots, X_{n}^{\alpha}\right)$ and $\left(T f^{*}\left(X_{1}, \ldots, X_{n}\right)\right)^{\beta}=f^{*}\left(X_{1}^{\beta}, \ldots, X_{n}^{\beta}\right)$, using the inclusivity of the interval extension $f^{*}([17$, Theorem 3.2.4]), it follows that: $f^{*}\left(X_{1}^{\alpha}, \ldots, X_{n}^{\alpha}\right) \subseteq f^{*}\left(X_{1}^{\beta}, \ldots, X_{n}^{\beta}\right)$ that is, $\left(T f^{*}\left(X_{1}, \ldots, X_{n}\right)\right)^{\alpha} \subseteq\left(T f^{*}\left(X_{1}, \ldots, X_{n}\right)\right)^{\beta}$.

Definition 15 If $X_{1}, X_{2}, Z \in T^{*}(\mathbb{R})$ and $*$ is a fuzzy operator, then $Z$ is semantically compatible with $X_{1} * X_{2}$, if $X_{1} * X_{2} \subseteq Z$; where we assume that this inclusion refers to the modal inclusion given in Definition 6. 
Theorem 16 (*-semantic theorem) Let $T f^{*}(X)$ be the $*$-fuzzy extension of a real continuous function $f$, above the vector $X=\left(X_{1}, \ldots, X_{n}\right)$, whose components are quantified trapezoidal fuzzy numbers. If $(Z, Q)$ is a quantified trapezoidal fuzzy number semantically compatible with $T f^{*}\left(X_{1}, \ldots, X_{n}\right)$, then $\forall \alpha \in[0,1]$ :

$$
\left(\forall x_{p} \in \operatorname{set}\left(X_{p}^{\alpha}\right)\right)\left(Q z \in \operatorname{set}\left(Z^{\alpha}\right)\right)\left(\exists x_{i} \in \operatorname{set}\left(X_{i}^{\alpha}\right)\right)
$$

such that $z=f\left(x_{p}, x_{i}\right)$, where $\left(X_{p}, X_{i}\right)$ are the components of $X$ split into its proper components $X_{p}$ and its improper components $X_{i}$.

Proof. Given $\alpha \in[0,1], f^{*}\left(X^{\alpha}\right)=\left(T f^{*}(X)\right)^{\alpha}$.

If $(Z, Q)$ is semantically compatible with $T f^{*}(X)$, then $f^{*}\left(X^{\alpha}\right)=\left(T f^{*}(X)\right)^{\alpha} \subseteq$ $Z^{\alpha}$ and we can apply the $*$-semantic interval theorem ([17, Theorem 3.3.1]), thereby obtaining the desired result.

The exact value $f^{*}(X)$ cannot be evaluated easily and it is common to replace every real operator in the function $f$ by its appropriate modal interval extension, as in classical interval analysis. This yields a result that includes the exact value $f^{*}(X)$.

This result implies, in general, a loss of information; but guarantees the applicability of the $*$-semantic theorem.

\section{Applications and numerical examples}

This section contains some applications of quantified trapezoidal fuzzy numbers. The first is detailed in Examples 1 and 2, which refers to the resolution of equations, showing that quantified trapezoidal fuzzy numbers can be used to solve equations which have no solution in the set of traditional trapezoidal fuzzy numbers.

The other applications show the applicability of quantified trapezoidal fuzzy numbers in financial calculations (Example 3) and to optics (Example 4).

In all these applications, we have emphasized the semantic interpretation of the results.

Example 1 In this first example we describe a typical situation in which a trivial fuzzy equation $A+X=B$ has no solution in the traditional set of fuzzy numbers. We find a solution of the equation using the extension of quantified trapezoidal fuzzy numbers presented in this paper and we also offer a correct semantic interpretation of the equation.

Let us consider the trapezoidal fuzzy equation $(2,5,7,9)+X=(6,7,8,9)$. There is no traditional fuzzy number $X$ which is a solution of the equation. However, using quantified trapezoidal fuzzy numbers and the dual operator, it holds that:

$$
X=(6,7,8,9)-\operatorname{dual}(2,5,7,9)=(4,2,1,0)
$$

The solution $X=(4,2,1,0)$ is an improper trapezoidal fuzzy number. Moreover, applying the semantic Theorem 16, we not only obtain the solution, but we can also give its semantic interpretation. Thus, it follows $\forall \alpha \in[0,1]$, that:

$$
\left(\forall a \in A^{\alpha}\right)\left(\exists b \in B^{\alpha}\right)\left(\exists x \in X^{\alpha}\right) \text { such that } a+x=b .
$$


Example 2 This second example solves and interprets the equation $A+X=B$, in which $A$ and $B$ are improper trapezoidal fuzzy numbers

$$
(8,4,2,0)+X=(11,8,7,10)
$$

whose solution $X=B-\operatorname{dual}(A)$ is $X=(3,4,5,10)$ : a proper trapezoidal fuzzy number.

The semantic interpretation (Theorem 16) of the equation is $\forall \alpha \in[0,1]$ :

$$
\left(\forall x \in X^{\alpha}\right)\left(\forall b \in B^{\alpha}\right)\left(\exists a \in A^{\alpha}\right) \text { such that } a+x=b .
$$

Example 3 This application is based in the calculus of the amount obtained by investing a quantity $C_{0}$ given by the proper trapezoidal fuzzy number:

$$
C_{0}=(10100,10150,10250,10400)
$$

in a deposit which has an interest rate $R$, represented by the proper trapezoidal fuzzy number:

$$
R=(0.02,0.024,0.026,0.03)
$$

for a period of time $T$, represented by the improper trapezoidal fuzzy number:

$$
T=(2.2,2.05,1.95,1.8) \text {. }
$$

The amount obtained is calculated using:

$$
C=C_{0} *(1+R * T) .
$$

When evaluating the expression $R * T$ it holds that:

$$
R * T=(0.02,0.024,0.026,0.03) *(2.2,2.05,1.95,1.8)
$$

and the fuzzy number obtained is not trapezoidal but must be converted to a quantified trapezoidal fuzzy number preserving the inclusion and minimizing the distance ([19]), to give a semantic interpretation (Theorem 16) of the final calculus. Thus, we obtain the proper trapezoidal fuzzy number

$$
(0.044,0.0492,0.05085,0.05415)
$$

Finally, the amount obtained is:

$$
\begin{aligned}
C= & (10100,10150,10250,10400) * \\
& (1+(0.044,0.0492,0.05085,0.05415)),
\end{aligned}
$$

which is not a trapezoidal fuzzy number (see Figure 5) and we will convert it to a quantified trapezoidal fuzzy number:

$$
C_{T}=(10544.335,10649.315,10771.2125,10963.16)
$$

(see Figure 6) which preserves inclusion and minimizes the distance to $C$. 


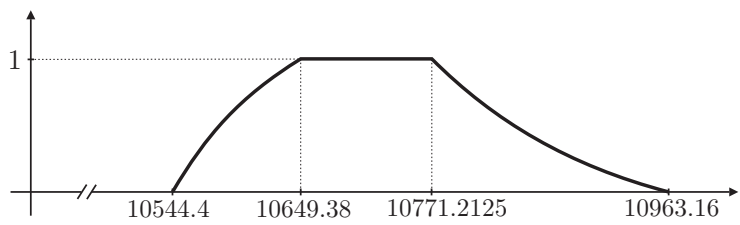

Figure 5: Representation of the fuzzy number $C$.

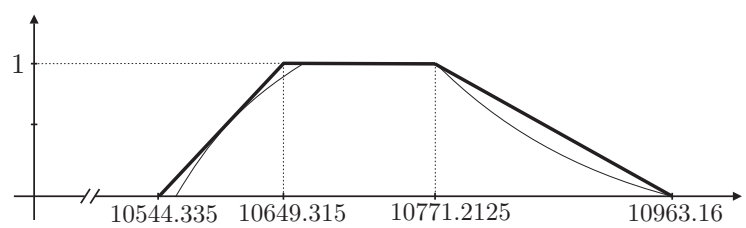

Figure 6: Representation of the trapezoidal fuzzy number $C_{T}$.

Thus,

$$
C_{0} *(1+r t) \subseteq C_{T}
$$

and applying the semantic theorem (Theorem 16) bearing in mind the modality of the initial values $C_{0}$ (proper), $R$ (proper), $T$ (improper) and the modality of the result $C$ (proper), we obtain the semantic interpretation $\forall \alpha \in[0,1]$ :

$$
\left(\forall c_{0} \in C_{0}^{\alpha}\right)\left(\forall r \in R^{\alpha}\right)\left(\exists c \in C_{T}^{\alpha}\right)\left(\exists t \in T^{\alpha}\right)
$$

such that $c=c_{0}(1+r t)$

Example 4 This last application is based in image formation by thin lenses. If the distances from the object to the lens and from the lens to the image are $d_{o}$ and $d_{i}$ respectively (see Figure 7), for a lens of negligible thickness, in air, the distances are related to the focal length $f$ by the thin lens formula:

$$
\frac{1}{d_{o}}+\frac{1}{d_{i}}=\frac{1}{f}
$$

Let us assume that any distance between 49 and 51 millimeters produces a correct vision and outside these margins, vision is less clear. We represent it by the improper trapezoidal fuzzy number

$$
D_{i}=(52,51,49,48) .
$$

The distance $d_{o}$ is represented by the proper fuzzy number

$$
D_{o}=(198,200,5000,5025) .
$$

When evaluating the focal length $F$ as a quantified trapezoidal fuzzy number using the expression

$$
F=\frac{1}{\frac{1}{D_{o}}+\frac{1}{D_{i}}},
$$




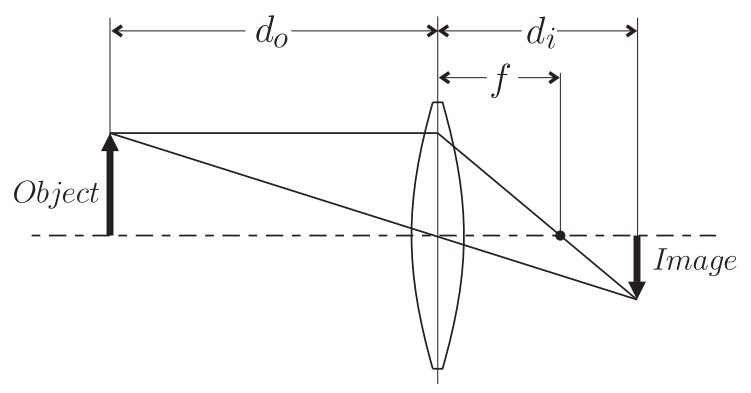

Figure 7: Ray diagram for converging convex lenses.

The partial results $\frac{1}{D_{o}}, \frac{1}{D_{i}}$ and $F$ are not trapezoidal fuzzy numbers and must be converted to quantified trapezoidal fuzzy numbers preserving the inclusion and minimizing the distance ([19]). Thus

$$
\begin{aligned}
\frac{1}{D_{o}} & \subseteq(0.000199,0.000199998,0.005,0.005050505) \\
\frac{1}{D_{i}} & \subseteq(0.0192289,0.0196059,0.0204082,0.020834)
\end{aligned}
$$

and finally we obtain

$$
F \subseteq(38.630824,39.354045,50.489771,51.472214)
$$

and applying the Semantic Theorem (Theorem 16), we obtain $\forall \alpha \in[0,1]$ :

$$
\left(\forall d_{o} \in D_{o}^{\alpha}\right)\left(\exists f \in F^{\alpha}\right)\left(\exists d_{i} \in D_{i}^{\alpha}\right)
$$

such that $f=\frac{1}{\frac{1}{d_{o}}+\frac{1}{d_{i}}}$

\section{Conclusions}

In this paper we have linked modal intervals with fuzzy numbers, devising a new structure of quantified trapezoidal fuzzy numbers, which extends the concept of fuzzy number in its classical or traditional sense. This not only provides new potential for the calculus, but also makes semantic sense of the calculations that are performed.

Quantified trapezoidal fuzzy numbers open the way to meaningful calculations that were excluded from the classical structure of fuzzy numbers. Thus, we open up new lines of research, such as the study of quantified fuzzy numbers without the trapezoidal restriction and the study of extended quantified trapezoidal fuzzy numbers, accepting that the core and the support of a quantified trapezoidal fuzzy number have not the same modality, thus there will be the possibility to consider distinct modalities for different $\alpha$-cuts of the same fuzzy number. Moreover, it will be possible to extend the semantic interpretation of a calculus using these modal fuzzy numbers efficiently. 


\section{References}

[1] S. Abbasbandy and B. Asadi, The nearest trapezoidal fuzzy number to a fuzzy quantity, Applied Mathematics and Computation 156 (2004), 381-386.

[2] R. Adillon and L. Jorba, A new point of view for fuzzy numbers and their defuzzification, International Journal of Uncertainty, Fuzziness and Knowledge-Based systems 23, No. 6 (2015), 909-926.

[3] G. Alefeld and J. Herzberger, Introduction to interval computations, Academic, New York, 1983.

[4] A.I. Ban, L. Coroianu and A. Khastan, Conditioned weighted L-R approximations of fuzzy numbers, Fuzzy Sets and Systems 283 (2016), 56-82.

[5] D. Dubois, H. Prade, Operations on fuzzy numbers, International Journal Systems Science 9 (1978), 613-626.

[6] R. Eslamipoor, H. Hosseini-nasab and A. Sepehriar, An improved ranking method for generalized fuzzy numbers based on Euclidian distance concept, Afrika Matematika 26 (2015) 1291-1297.

[7] E. Gardeñes, H. Mielgo, Modal intervals: functions, in: Polish Symposium on Interval and Fuzzy Mathematics, Poznan, Poland, 1986.

[8] E. Gardeñes, H. Mielgo and A. Trepat, Modal intervals: reasons and ground semantics, Interval Mathematics. Lecture Notes in Computer Science 212 (1986), Springer Heildelberg, 27-35.

[9] E. Gardeñes et al., Modal intervals, Reliable computing 7 (2001), 77-111.

[10] P. Grzegorzewski and E. Mrówka, Trapezoidal approximation of fuzzy numbersrevisited, Fuzzy Sets and Systems 158 (2007), 757-768.

[11] P. Grzegorzewski, Trapezoidal approximations of fuzzy numbers preserving the expected interval - Algorithms and properties, Fuzzy Sets and Systems 159 (2008), 1354-1364.

[12] M. Janizade-Haji, H.K. Zare, R. Eslamipoor and A. Sepehriar, A developed distance method for ranking Generalized fuzzy numbers, Neural Computing and Applications 25 (2014), 727-731.

[13] E. Kaucher, Algebraische erweiterungen der intervallrechnung unter erhaltung der ordnungs und verbandsstrukturen, Computing Supplementum 1 (1977), 65-79.

[14] R.E. Moore, Interval analysis, Englewood: Prentice Hall, 1966.

[15] K. Nickel, Verbandtheoretische grundlagen der intervallmathematik, Lecture Notes in Computer Science 29 (1975), 251-262.

[16] Z. Pawlak, Rough sets, International Journal of Computer and Information Sciences 11 (1982), 341-356. 
[17] M. Sainz, J. Armengol, R. Calm, P. Herrero, L. Jorba and J. Vehi, Modal Interval Analysis: New Tools for Numerical Information, Lecture Notes in Mathematics 2091 (2014), Springer, Switzerland.

[18] C. Veeramani, C. Duraisami and M. Sumathi, Nearest Symmetric Trapezoidal Fuzzy Number approximation preserving expected interval, International Journal of Uncertainty, Fuzziness and Knowledge-Based Systems 21, No. 5 (2013), 777794.

[19] M. Wagenknecht and V. Schneider, Inclusion of fuzzy numbers. The variable Plateau Case, in: EUSFLAT - LFA, 2005.

[20] Y.Y. Yao, A comparative study of fuzzy sets and rough sets, Information Sciences 109 (1998) 227-242.

[21] C. T. Yeh, A note on trapezoidal approximations of fuzzy numbers, Fuzzy Sets and Systems 158 (2007), 747-754.

[22] C. T. Yeh, Trapezoidal and triangular approximations preserving the expected interval, Fuzzy Sets and Systems 159 (2008), 1345-1353.

[23] L.A. Zadeh, Fuzzy sets, Information and Control 8 (1965), 338-353.

[24] J. Zhan, Q. Liu, B. Davvaz, A new rough set theory: rough soft hemirings, Journal of Intelligent and Fuzzy System 28 (2015), 1687-1697. 